\title{
La participación de los millennials en el marco de la democracia representativa en Perú durante el 2019
}

\section{The participation of millennials in the framework of representative democracy in Peru during 2019}

\author{
Odar Alexander Carranza Reyes* \\ Martha Alicia Romero Echevarría** \\ Facultad de Derecho, \\ Universidad de San Martín de Porres, Perú
}

\section{Resumen}

La participación masiva de los jóvenes respecto a los sucesos políticos estuvo aquietada por muchos años; sin embargo, con el posicionamiento de las comunicaciones a nivel global, la generación millennial se va involucrando cada vez más. Es así que en el marco de la democracia representativa de develar una participación cada vez más activa, ahora los jóvenes comunican su sentir y su postura, mostrando su conformidad o disconformidad con las decisiones y acontecimientos políticos. Partiendo de esta premisa, y con un enfoque mixto, se aplicó un cuestionario de 16 preguntas a 3474 millennials, estudiantes de institutos superiores y tecnológicos ubicados en Lima; además, se realizó un análisis de las declaraciones de académicos y jefes de Estado, así como de casos internacionales donde se destaca la participación de esta generación, como aconteció en la Primavera Árabe y en otros sucesos ocurridos en Sudamérica, para vislumbrar las brechas que se abren en la estructura política del Perú.

Palabras clave: generación millennial, democracia representativa, legalidad, legitimidad.

Este es un artículo Open Access bajo la licencia Creative 


\begin{abstract}
The massive participation of young people regarding political events was muted for many years. However, with the positioning of communications globally, the millennial generation is becoming more and more involved. Thus, in the framework of representative democracy of revealing an increasingly active participation, young people now communicate their feelings and their position, showing their agreement or disagreement with political decisions and events. Starting from this premise, and with a mixed approach, a questionnaire of 16 questions was applied to 3474 millennials, students of higher and technological institutes located in Lima. Furthermore, an analysis was carried out of the statements of academics and heads of state, as well as international cases where the participation of this generation stands out, as happened in the Arab Spring and in other events that occurred in South America, to glimpse the gaps that are open in the political structure of Peru.
\end{abstract}

Keywords: millennial generation, representative democracy, legality, legitimacy.

\title{
Introducción
}

Diversos acontecimientos de la historia han modificado el curso de la humanidad, determinando cambios y posturas en las personas, y consecuentemente en las sociedades. La llegada de Internet representó la masificación de la comunicación y la proliferación de recursos informativos, el mundo quedó conectado y las innovaciones tecnológicas son cada vez más accesibles.

Con el empoderamiento de las tecnologías de la comunicación e información (TIC) se pone de manifiesto, dentro del sistema de democracia representativa, un escenario político que apenas se empieza a vincular con los avances tecnológicos, quedando los millenials enlazados a un gobierno estadual mediante los recursos digitales, a pesar de que no son ajenos a ellos, ya que en su mayoría se trata de nativos digitales (Carranza, 2019). 
El impresionante desarrollo de la tecnología se refleja en la existencia actual de más de un equipo móvil por cada habitante del mundo, tendencia imparable marcada por un crecimiento exponencial, que se puede visualizar en la página de Global System for Mobile communications (GSM, s.f.), permitiéndonos calcular que, para el 2022, los dispositivos duplicarán el número de habitantes.

El escenario actual muestra que la participación de la ciudadanía en política y en hechos sociales es cada vez más cercana, en especial para los millennials. En este proceso se construyen mecanismos para que los gobiernos ocupen un espacio visible a través de la tecnología, presionando al establishment para que evalúe una redimensión (Misses-Liwerant, 2016).

Existen pocos estudios acerca de las características de la generación que nos ocupa en cuanto a su participación en la vida política empleando la comunicación instantánea por las redes, con manifestaciones de diversa índole, que van desde un «meme» hasta un pronunciamiento consistente en busca del equilibrio social en el ámbito de la democracia representativa (Molinari, 2011).

En la Universidad Complutense de Madrid, Almeida (2015) presentó un estudio acerca del impacto de la tecnología en la construcción de una ciudadanía plena, proponiendo el término Homo comunicantis para describir al individuo del siglo XXI con una participación activa en la Ciudad Global, desarrollando su potencial cívico y aportando en la toma de decisiones, configurando el perfil del nuevo ciudadano global.

Más adelante, Enríquez (2019) investigó la participación de los nuevos movimientos sociales en los principales sucesos ocurridos entre el 2008 y el 2015 a raíz de la caída del muro de Berlín, mostrando que las TIC se constituyeron en una plataforma activa que impulsó la intervención de personas que se solidarizaron -e incluso de personajes del mundo- en una nueva forma de organización colectiva.

En poco tiempo la generación Baby Boomer y la generación $X$ serán reemplazadas, y la conducción de todo lo que ocurra en el mundo será asumida por la generación millennial, tomando postura ante un sistema 
político de representación que está en contra de su tiempo y de sus competencias digitales, redimensionando la democracia representativa mutatis mutandis en el contexto digital para hacer frente a las exigencias de la vida pública.

Con el tiempo han ido mejorando los procesos, de manera que en la actualidad se ha dinamizado la participación en redes. Por ello se consideró necesario analizar el impacto de esta participación, en particular de índole política, de la generación millennial, al estar integrada por personas hiperconectadas que se muestran críticas ante los acontecimientos sociales y que no se conforman con la información recibida, cuestionándola en busca de respuestas satisfactorias, además de fluir en las redes y formar comunidades digitales.

\section{El uso de las TIC por los millennials}

Toda la información transmitida por las redes se caracteriza por promover la comunicación interactiva y los mass media son parte de esta digitalización, haciendo posible un intercambio de información que cobra gran importancia por la interacción que tiene. Las personas ya no solo consumen noticias, sino que también las movilizan al compartirlas, comentarlas, criticarlas o reaccionar ante ellas.

Los peruanos tenemos la oportunidad de ser parte de esta interconexión y de allí surge la necesidad de masificar el uso de Internet en todos los espacios; sin embargo, aún existen lugares con conexiones malas o simplemente inexistentes. En el mismo sentido, se constata que hay un sector de la población que no cuenta con los dispositivos para conectarse (Romero y Estrada, 2020).

En el 2016 se conformó en el Perú una comisión multisectorial temporal, con la finalidad de elaborar un plan nacional para el desarrollo de la banda ancha y que sirviera como marco de referencia. Dicha medida no ha tenido el impacto esperado ya que la masificación de Internet a través de esta modalidad no solo depende del tendido de una red principal. En este sentido, es necesario que el Estado involucre a los gestores públicos capacitados para hacer las coordinaciones a nivel multisectorial. 
La comunicación multimedia proporciona los mecanismos para transmitir mensajes claros y precisos, incorporando un lenguaje digital y relatos que son adecuados a los múltiples medios, usando los diferentes recursos que ofrece la Web 2.0, de modo que el consumidor es ahora un prosumidor. De esta manera, los millennials se convierten en cocreadores de contenidos, asumiendo una participación activa en los espacios digitales.

La multimedialidad permite que se imponga una convergencia mediática por la manera en que confluyen los distintos medios y plataformas. Según Pérez y Acosta (2003), dicha convergencia le brinda soporte a la industria informativa y comunicacional -medios escritos, televisivos y audiovisuales-, aprovechando los recursos tecnológicos que están a disposición de las personas, para que encuentren la multiplataforma con la que se sientan más cómodas al interactuar.

La accesibilidad a los medios digitales permite que el proceso comunicativo sea eficiente y que los contenidos se revistan de mayor sentido. Guenaga et al. (2017) sostienen que las pautas recomendadas para diseñar y desarrollar tanto las aplicaciones como los contenidos están concebidas para que el producto sea accesible al consumidor y que pueda transmitirse de manera fácil en los distintos canales.

Las TIC generan una cuantiosa información que ha cambiado la forma de comprender los sucesos, facilitando la toma de decisiones. La Big Data se encarga de examinar y reproducir dicha información en grandes repositorios, donde una incalculable cantidad de datos está a disposición de los usuarios (Salazar, 2016, p. 4). En este caso, han servido para hacer un análisis de la percepción que tienen los millennials acerca de la cantidad y calidad de su participación en el quehacer político.

\section{Participación cívica o ciudadana}

Los ciudadanos son cada vez más activos, participan en las decisiones que toma el gobierno utilizando los canales digitales, sin ser necesariamente servidores públicos o militantes de algún partido político, de manera que lo hacen de forma independiente. Guamán (2017) opina que los ciudadanos comprometidos contribuyen con sus manifestaciones a lograr el equilibrio en las decisiones, debiendo ser vigilantes y críticos con la gestión pública. 
En ese sentido, los ciudadanos digitales pueden y deben propiciar la participación masiva para exigir mejoras en busca del bienestar social. La presión que se ejerce utilizando los medios digitales servirá para reducir las decisiones que contravengan sus derechos, encausando el fortalecimiento de la niñez, adolescencia y juventud.

\section{Millennials y la democracia representativa}

Los ciudadanos de la generación millennial son conocidos como nativos digitales. Al ser tecnológicos manejan con facilidad varios dispositivos y canales de manera simultánea. Su característica principal es que están conectados permanentemente, por lo que requieren de contenido proveniente de las redes y de las otras plataformas, fortaleciendo las comunidades digitales.

Paredes (2020) analiza las características de los millennials en torno al quehacer de los políticos en las redes sociales y la forma en que se acercan a las personas, destacando la conformación de las comunidades que vinculan la política con el mundo digital de accesibilidad e interacción con la comunicación.

Howe y Strauss (2000), como se citó en Gutiérrez y Posada (2018), acuñan el término de millennials rising, describiéndolos como «optimistas, positivos, alegres, jugadores de equipo, cooperativos, inteligentes, seguidores de reglas» (p. 2). Se caracterizan por estar acostumbrados al uso de las tecnologías.

Se trata de una generación que creció en una época de crisis, en la que a pesar de una progresión positiva en la economía nacional no se ha logrado el equilibrio social. En este sentido, Jasso-Peña et al. (2019) acotan que los millennials encontraron en la tecnología el recurso para participar activamente en el quehacer local, nacional y mundial.

El concepto de democracia representativa ha ido evolucionando explicando la crisis de representación de los países donde se construyó el ideal del interés común. Con el uso de las redes sociales se articularon «nuevas formas de expresión en la dimensión actual de opinión pública política autónoma» (Martino, 2018, p. 261), haciendo alusión a la preocupación por la constante participación y control que ejercen en sus representantes, llegando incluso a una confrontación. 
Desde el Congreso de la República del Perú, Guembes (2015) expresa que parece existir una fuerte capacidad y voluntad para autogobernarnos con el apoyo y las facilidades provistas por las TIC. Aun así, existe la necesidad de que los políticos se encarguen de ordenar los asuntos que beneficien a la mayoría de los ciudadanos.

En el 2019 el mundo estuvo expuesto a las acciones y reacciones de movimientos sociales que surgieron como protesta ante alguna decisión política ocurrida en sus espacios, llegando a activar estados de emergencia en países como Hong Kong, Ecuador, Chile o Colombia. Cabe enfatizar que la mayoría de los manifestantes estuvo conformada por millennials que se conectaron usando las TIC con el fin de influir o cambiar el establishment de sus países.

La primera convocatoria virtual de los millennials peruanos ocurrió a raíz del caso «La Repartija», ocurrido en julio del 2013. Se produjo un movimiento disruptivo que ocasionó una crisis política de tal magnitud que ocasionó cambios e hizo retroceder a los políticos que estaban implicados en el asunto.

El principio de legalidad exige que el Estado se alinee al cumplimiento de la ley, tomando acciones que sean validadas por la sociedad de acuerdo con los preceptos, quedando establecido que ningún accionar desde cualquier espacio del Estado puede afectar o extinguir los derechos de los ciudadanos, como está plasmado en el artículo $150^{\circ}$ de la Constitución Política peruana.

El objetivo del presente trabajo es analizar la participación de los millennials en las comunidades virtuales mediante el uso de las TIC en el marco de la democracia representativa, tomando como referencia los hallazgos del estudio realizado por Carranza (2019).

\section{Método}

La investigación es de diseño no experimental y de nivel descriptivoexplicativo (Hernández et al., 2014). Participaron 3474 millennials, estudiantes de nivel superior en institutos y universidades de Lima, cuyas edades oscilan entre los 18 y 35 años, pertenecientes a los tres estratos socioeconómicos establecidos por el Instituto Nacional de Estadística e Informática (INEI). Carranza (2019) les aplicó un cuestionario de 16 preguntas con el fin de conocer sus opiniones, a la vez de establecer su nivel socioeconómico. 


\section{Resultados}

De las respuestas recolectadas en cuanto al uso de las TIC (ver Tabla 1), se deduce que los millennials prefieren el WhatsApp como el principal medio de comunicación por su accesibilidad e interactividad; por su facilidad para enviar y recibir mensajes personales y grupales; así como por las llamadas y videollamadas que permiten conectarse con personas que se encuentran en cualquier parte del mundo a un costo mínimo.

En cuanto al uso de las distintas redes sociales, los resultados muestran que Facebook e Instagram son visitadas aproximadamente 10 veces al día, distinguiéndose el uso de Twitter entre los millennials que pertenecen a los segmentos A y C (también con 10 visitas diarias), mientras que el segmento $B$ duplica la cantidad indicada. En la mayoría de manifestaciones públicas ocurridas alrededor del mundo, las redes sociales han servido para interactuar compartiendo información social y política, generando opiniones y decisiones en el ciudadano digital.

La plataforma virtual de preferencia entre los millennials es YouTube. Carranza (2019) observa que tiene un acceso muy alto en los tres segmentos sociales, permitiendo ver y crear contenidos audiovisuales, siendo una gran fuente de información para aprender y ser críticos; por eso contrastan y complementan la información si les interesa.

En relación a los contenidos que son de interés para los millennials (ver Tabla 2), la mayoría prefiere ver publicidad por las redes sociales, al tiempo que se enteran de las noticias nacionales e internacionales usando los diferentes medios digitales.

El trabajo realizado por Carranza (2019) recogió información acerca de la opinión que tienen los millennials sobre sus autoridades, comenzando por el sector político, encontrando que la mayoría opina que prima la corrupción y que hay poca o ninguna transparencia en la gestión que realizan, por lo que no se sienten representados por ellas. Declaran asimismo que es importante y necesario que se transparente la información en torno a todas las acciones políticas, pudiendo tener como aliados de la transparencia en la gestión a Facebook e Instagram, e incluso a YouTube, para que de manera visual 
muestren las evidencias y den a conocer los hechos, acontecimientos y decisiones políticas y públicas.

\section{Tabla 1}

Uso de las TIC por los millennials

\begin{tabular}{|c|c|c|c|c|c|c|c|}
\hline TIC utilizada & Seg. A & Seg. B & Seg. C & Visitas a Instagram & Seg. A & Seg. B & Seg. C \\
\hline Twitter & 261 & 289 & 278 & 1 a 10 veces al día & 363 & 629 & 428 \\
\hline Facebook & 774 & 914 & 799 & 11 a 20 veces al día & 123 & 247 & 169 \\
\hline Instagram & 717 & 1127 & 824 & 20 a 30 veces al día & 129 & 166 & 137 \\
\hline WhatsApp & 873 & 1296 & 996 & \multirow{3}{*}{$\begin{array}{l}\text { Frecuencia de uso } \\
\text { de WhatsApp }\end{array}$} & \multirow{3}{*}{ Seg. A } & \multirow{3}{*}{ Seg. B } & \multirow{3}{*}{ Seg. C } \\
\hline YouTube & 555 & 889 & 897 & & & & \\
\hline Messenger & 321 & 431 & 357 & & & & \\
\hline Otras & 33 & 43 & 38 & \multirow{3}{*}{$\begin{array}{l}1 \text { a } 10 \\
11 \text { a } 20 \\
20 \text { a } 30\end{array}$} & \multirow{3}{*}{$\begin{array}{c}57 \\
516 \\
123\end{array}$} & \multirow{3}{*}{$\begin{array}{c}88 \\
723 \\
321\end{array}$} & \multirow{3}{*}{$\begin{array}{c}69 \\
511 \\
263\end{array}$} \\
\hline & & & & & & & \\
\hline Frecuencıa dıarıa & Seg. A & Seg. B & Seg. C & & & & \\
\hline 1 a 3 horas & 231 & 299 & 274 & \multirow{2}{*}{ Visitas a YouTube } & \multirow[t]{2}{*}{ Seg. A } & \multirow[t]{2}{*}{ Seg. B } & \multirow[t]{2}{*}{ Seg. $C$} \\
\hline 3 a 6 horas & 366 & 587 & 489 & & & & \\
\hline 6 a 9 horas & 159 & 198 & 141 & \multirow{3}{*}{$\begin{array}{l}1 \text { a } 10 \\
11 \text { a } 20 \\
20 \text { a } 30\end{array}$} & \multirow{3}{*}{$\begin{array}{c}346 \\
79 \\
58\end{array}$} & \multirow{3}{*}{$\begin{array}{l}487 \\
179 \\
125\end{array}$} & \multirow{3}{*}{$\begin{array}{l}491 \\
181 \\
131\end{array}$} \\
\hline & & & & & & & \\
\hline VIsitas a IWItter & Seg. A & Seg. B & Seg. C & & & & \\
\hline 1 a 10 veces al día & 106 & 85 & 107 & \multirow{2}{*}{ Uso de Messenger } & \multirow[t]{2}{*}{ Seg. A } & \multirow{2}{*}{ Seg. B } & \multirow[t]{2}{*}{ Seg. C } \\
\hline 11 a 20 veces al día & 91 & 109 & 83 & & & & \\
\hline 20 a 30 veces al día & 30 & 41 & 53 & \multirow{3}{*}{$\begin{array}{l}1 \text { a } 10 \\
11 \text { a } 20 \\
20 \text { a } 30\end{array}$} & \multirow{3}{*}{$\begin{array}{l}119 \\
69 \\
73\end{array}$} & \multirow{3}{*}{$\begin{array}{l}189 \\
111 \\
89\end{array}$} & \multirow{3}{*}{$\begin{array}{c}161 \\
84 \\
74\end{array}$} \\
\hline Visitas a Facebook & Seg. A & Seg. B & Seg. C & & & & \\
\hline 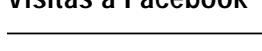 & seg. A & seg. D & seg. $C$ & & & & \\
\hline 1 a 10 veces al día & 438 & 367 & 391 & & & & \\
\hline 11 a 20 veces al día & 214 & 284 & 269 & & & & \\
\hline 20 a 30 veces al día & 113 & 239 & 104 & & & & \\
\hline
\end{tabular}


Tabla 2

Resultados de los contenidos que son de interés para los millennials

\begin{tabular}{|c|c|c|c|c|c|c|c|}
\hline $\begin{array}{l}\text { Ve publicidad en } \\
\text { redes sociales }\end{array}$ & Seg. A & Seg. B & Seg. C & $\begin{array}{l}\text { Se debe informar } \\
\text { la labor política }\end{array}$ & Seg. A & Seg. B & Seg. C \\
\hline Sí & 717 & 1017 & 923 & Sí & 939 & 1351 & 1167 \\
\hline No & 231 & 339 & 247 & No & 9 & 4 & 3 \\
\hline $\begin{array}{l}\text { Ve noticias } \\
\text { nacionales }\end{array}$ & Seg. A & Seg. B & Seg. C & $\begin{array}{l}\text { Redes que le sirven } \\
\text { para informarse }\end{array}$ & Seg. A & Seg. B & Seg. C \\
\hline Sí & 773 & 1123 & 937 & Twitter & 231 & 277 & 254 \\
\hline Nno & 175 & 233 & 233 & Facebook & 789 & 952 & 811 \\
\hline $\begin{array}{l}\text { Ve noticias } \\
\text { internacionales }\end{array}$ & Seg. A & Seg. B & Seg. C & $\begin{array}{l}\text { Instagram } \\
\text { WhatsApp }\end{array}$ & $\begin{array}{l}726 \\
633\end{array}$ & $\begin{array}{l}876 \\
749\end{array}$ & $\begin{array}{l}793 \\
671\end{array}$ \\
\hline Sí & 819 & 1211 & 941 & $\begin{array}{l}\text { Youlube } \\
\text { Messenger }\end{array}$ & $\begin{array}{l}891 \\
213\end{array}$ & $\begin{array}{c}1295 \\
314\end{array}$ & $\begin{array}{l}1148 \\
194\end{array}$ \\
\hline No & 129 & 145 & 229 & Información política & Seg. A & Seg. B & Seg. C \\
\hline $\begin{array}{l}\text { Opinión acerca de la } \\
\text { política nacional }\end{array}$ & Seg. A & Seg. B & Seg. C & debe ser & 513 & 726 & 642 \\
\hline Es corrupta & 568 & 847 & 691 & Disponible & 337 & 453 & 376 \\
\hline Poca transparencia & 311 & 403 & 377 & Propuestas claras & 65 & 123 & 109 \\
\hline Inestable & 39 & 72 & 66 & Otras & 33 & 54 & 43 \\
\hline Decadente & 11 & 12 & 8 & & & & \\
\hline En crisis & 9 & 8 & 7 & & & & \\
\hline Otras opiniones & 10 & 14 & 21 & & & & \\
\hline \multicolumn{2}{|c|}{$\begin{array}{l}\text { Se siente representado Seg. A } \\
\text { por sus políticos }\end{array}$} & Seg. B & Seg. C & & & & \\
\hline Sí & 13 & 7 & 4 & & & & \\
\hline No & 935 & 1349 & 1166 & & & & \\
\hline
\end{tabular}




\section{Discusión}

El estudio demuestra que los millennials que cursan estudios superiores en Lima tienen preferencia por el uso de las TIC y permanecen conectados la mayor cantidad del tiempo, demandando que la información por redes sociales sea clara, lo que se ajusta a las características de esta generación de nativos digitales.

Para ellos las TIC son una fuente inagotable de interacción con los contenidos, que les permiten formar opinión y tomar decisiones para actuar frente a los acontecimientos que atentan contra la dignidad, convirtiendo a los millennials en ciudadanos digitales dispuestos a iniciar una protesta que rápidamente pone en alerta a los demás.

La preferencia en cantidad y calidad de información por parte de los millennials es privilegio de Internet, que les permite navegar alrededor del mundo y conectarse con otros espacios, pudiendo formar redes de contacto virtual, dar a conocer sus opiniones y puntos de vista $y$, al encontrarse en desacuerdo, sumarse a las protestas constituyéndose en torno a la democracia participativa.

Cabe destacar que en el marco de esta investigación se navegó por Internet con el fin de obtener información digital acerca de la transparencia de los políticos, de sus actividades y de las decisiones que afectan la calidad de la vida del ciudadano, pero los esfuerzos fueron en vano: solo se encuentra alguna hoja de vida y escasas notas en los medios de comunicación que, por supuesto, destacan la peor parte de la política y de los políticos.

\section{Conclusiones}

La generación millennial se vincula con el quehacer social y político a nivel nacional y mundial por medio de las TIC, inspirándose en la legitimación como modelo para actuar en favor del bien social.

Los medios de comunicación virtual proveen un espacio de información permanente para los millennials, quienes interactúan todo el tiempo 
mostrando su postura y compartiendo sus opiniones, de manera que se construyen redes virtuales a nivel global en cuestión de minutos.

Con el estudio se ha determinado que los millennials exigen calidad y claridad en la información, propiciando escenarios de democracia representativa que afiancen la participación de los ciudadanos, con el fin de disminuir el grado de insatisfacción generado por la falta de estrategias para comunicar con transparencia.

\section{Referencias}

Almeida, M. T. de. (2015). Comunicación digital, educación y ciudadanía global: un nuevo paradigma [Tesis de grado. Universidad Complutense de Madrid]. https://dialnet.unirioja.es/servlet/tesis?codigo=100412

Carranza, O. A. (2019). El impacto de las tecnologías de la información y la comunicación en la generación millennial en el marco de la democracia representativa [Tesis de maestría, Universidad de San Martín de Porres]. https://repositorio.usmp.edu.pe/handle/20.500.12727/5805

Enríquez, J. A. (2019). La comunicación digital en los nuevos movimientos sociales (2008-2015) [Tesis doctoral. Universidad Complutense de Madrid]. https://dialnet.unirioja.es/servlet/tesis?codigo=230020

Global System for Mobile communications - GSM. (s.f.). Representing the worldwide mobile communications industry. https://www.gsma.com/

Guamán, E. V. (2017). La participación ciudadana en la gestión del Gobierno Autónomo Descentralizado del Cantón Paltas, período 2015 [Tesis de grado. Universidad Nacional de Loja]. http://repositorio.ucv.edu.pe/bitstream/ handle/UCV/15319/Mestanza_CJA.pdf?sequence=1\&isAllowed=y

Guembes, C. (2015). Olvido constitucional y vacío representativo en el Perú. Fondo Editorial del Congreso del Perú. https://www2.congreso.gob.pe/Sicr/FondoEditorial/SIFonEdi.nsf/CategWebUPCongTodos/ 5FCOBA0AAF911373052586A3005235C8?opendocument

Guenaga, M. L., Barbier, A. y Eguíluz, A. (2017). La accesibilidad y las tecnologías en la información y la comunicación. TRANS. Revista de Traductología, 11, 155-169. http://www.revistas.uma.es/index.php/trans/ article/view/3104/2867

Gutiérrez, C. P. y Posada, L. P. (2018). Caracterización del comportamiento de los millennials: una revisión de literatura. (Doctoral Dissertation, Universidad EAFIT). https://repository.eafit.edu.co/handle/10784/12658

Hernández, R., Fernández, C. y Baptista, P. (2014). Metodología de la investigación científica. Mc Graw Hill.

Jasso-Peña, F. de J., Gudiño-Paredes, S. y Tamez-Solís, J. P. (2019). Centennials, ciudadanos globales y digitales. Praxis, 15(1), 11-23. http://revistas.unimagdalena.edu.co/index.php/praxis/article/view/2981

Martino, A. A. (2018). Crisis de la democracia representativa: alternativas participativas o democracia directa con medios electrónicos (Crisis of representative democracy: participatory alternatives or direct democracy with electronic media). Eunomía. Revista en Cultura de la Legalidad,14, 9-32.

Misses-Liwerant, J. B. (2016). Pensar a la sociedad y al espacio público: inclusión y democracia. Revista mexicana de ciencias políticas y sociales, 61(227), 9-30.

Molinari, P. (2011). Turbulencia generacional. Temas. http://ebookcentral.proquest.com/lib/bibliotecafmhsp/ detail.action?doclD=5487079 
Paredes, M. F. (2020). ¿Qué esperan los millennials de los políticos en redes sociales y cómo llamar su atención? GIGAPP Estudios / Working Papers, 7(166-182), 517-528.

Pérez, A. y Acosta, H. (2003). La convergencia mediática: un nuevo escenario para la gestión de información. ACIMED, 11(5). http://scielo.sld.cu/scielo.php?script=sci_arttext\&pid=S1024-94352003000500003\&lng=es\&tlng=es

Romero, M. A. y Estrada, M. J. (2020). El periodista emprendedor: asumiendo retos en la nueva normalidad. Cultura, 34, 89-103. https://doi.org/10.24265/cultura.2020.v34.07

Salazar, J. (2016). Big data en la educación. Revista Digital Universitaria, 17(1). http://www.revista.unam.mx/ vol.17/num1/art06/art06.pdf 